\title{
Minimum PER User-Energy Profile for Massive SIC Receivers under an Average Energy Constraint
}

\author{
Josep Sala-Alvarez, Francesc Rey, Javier Villares and Francesc Molina \\ Dept. of Signal Theory and Communications, Universitat Politècnica de Catalunya - Barcelona Tech (http://spcom.upc.edu) \\ c/ Jordi Girona 1-3, Campus Nord UPC, Edifici D5, 08034 Barcelona (Spain) \\ \{josep.sala,francesc.rey,javier.villares\}@upc.edu , fmolina@tsc.upc.edu
}

\begin{abstract}
In dense wireless networks (large number of users) and under high traffic loads, inter-user interference constitutes a factor limiting network's performance. Successive Interference Cancellation (SIC) has successfully counteracted this problem. Nonetheless, the aggregate Packet Error Rate (PER) when SIC is employed heavily depends on the users' symbol energies. We show that in a wireless (satellite) scenario where users share the same encoder, we may apply Variational Calculus (VC) in the largeuser limit to derive the optimum distribution of ordered energies, or Energy Profile, and its associated Signal to Interference plus Noise Power Ratio (SINR) over consecutive SIC stages when an average energy constraint is enforced. Both profiles are shown to solve a differential equation system and to be a non-increasing function of the user decoding order. A comparative analysis is carried out for two representative encoders.

Index Terms-Successive Interference Cancellation, Packet
\end{abstract} Error Rate, Satellite, Multiple Access, Variational Calculus

\section{INTRODUCTION}

Interference may pose a severe limitation to massive wireless multiple access unless the central receiver is endowed with strong multipacket reception capabilities. In that respect, Interference Cancellation (IC) has been extensively studied [1][2][3]. Such systems have been proposed for satellite communications, combining Direct Sequence (DS) Code Division Multiple Access (CDMA) with a SIC receiver [4][5][6] or using multipacket transmission as in Contention Resolution Diversity Slotted ALOHA (CRDSA) [7][8]. The use of powerful encoders coupled with power imbalance at reception favours IC and has considerably improved the throughput of such random access protocols [4][9]. In a context characterized by high user activity and spectrum/orbit congestion [10], it is of interest to develop schemes for space assets that optimize the aggregate spectral efficiency of the user population.

We consider a transparent satellite (i.e., operating as a repeater), in which the uplink received multiple access signal is transmitted to the ground gateway central receiver. The satellite power efficiency constitutes a relevant factor in that system optimization is carried out for a specific operating point (OP) of the on-board amplification chain, which can be defined in terms of the received power on the uplink. The uplink user channels are slowly time-varying, practically stationary over the packet duration and propagation delay and characterized by a strong line-of-sight component (e.g., fixed/mobile satellite services using medium-complexity stations/terminals equipped with a directive antenna). If a satellite-to-users downlink pilot signal is available, perfect channel gain information at transmission for all users is reasonably assumed. A proofof-concept analysis is performed in such case for users that share the same modulation/coding scheme and may adjust their transmission power. A practical encoder is adopted, and, to allow typical satellite terminal operation at a low peakto-average power ratio, a DS spread spectrum modulation is selected for uplink access. Under these conditions, we derive, for the specific SIC policy described in Section II and with affordable control overhead at system level, the user energy profile that optimizes a global utility (the aggregate PER) under an average received energy constraint related to the OP of the on-board amplification chain. With this goal in mind, we show that, for practical coding schemes, the optimum SINR profile is decreasing in the SIC decoding order when the system is heavily loaded. Similar results were obtained in [11] for a less powerful SIC policy that halts at the first detected packet loss. This conclusion contrasts with other previous results, for which the optimum SINR is uniform over consecutive SIC stages. Specifically, the uniform SINR profile is found when the objective is to optimize the asymptotic capacity [12], when the average energy limitation is substituted by a given power imbalance constraint (maximum to minimum of the user energy profile) [13], or when an iterative SIC receiver is implemented (assuming some heuristic simplifications [14]).

Section II presents the system model and the differential equation for the SIC receiver in the large-user regime [13][15]. Sec. III describes the spectral efficiency optimization problem and the computation of the corresponding SINR and energy profiles. Simulations for two coding schemes are shown in Sec. IV, with conclusions in Sec. V. Secs. VI and VII support the corresponding $\mathrm{VC}$ derivations.

\section{SYSTEM MODEL}

We adopt a DS spread-spectrum model in which $K$ users transmit on the uplink with symbol energy $E_{\mathrm{x}}[k]$, with $1 \leq k \leq K$ the user index. The complex equivalent baseband signal of the $k$-th user's $D$-symbol packet is given by,

$$
x_{k}(t)=A_{k} \sum_{n=0}^{D-1} \sum_{m=0}^{N-1} c_{k}[N n+m] a_{k}[n] p_{c}\left(t-(N n+m) T_{c}\right)
$$

with $A_{k}$ an amplitude factor for power control, $N$ the spreading factor, $c_{k}[N n+m]$ a long spreading code [16], $a_{k}[n]$ the symbol sequence, $p_{c}(t)$ the chip pulse and $T_{c}$ the chip period.

(c) 2017 IEEE. Personal use of this material is permitted. Permission from IEEE must be obtained for all other uses, in any current or future media, including reprinting/republishing this material for advertising or promotional purposes, creating new collective works, for resale or redistribution to servers or lists, or reuse of any copyrighted component of this work in other works. 
Under the flat fading model, the complex equivalent baseband signal received at the gateway after down-conversion is $y(t)=$ $\sum_{k=1}^{K} \beta_{k} x_{k}\left(t-\tau_{k}\right)+n(t)$, with $\beta_{k}$ and $\tau_{k}$ the respective $k$-th user's end-to-end complex channel and timing offset, and $n(t)$ additive white Gaussian noise. For the sake of meaningful and affordable theoretical analyses, carrier frequency offsets are obviated and perfect knowledge of user channel and symbol energy is assumed. At the gateway, users are received with symbol energy $E_{s}[k]=\left|\beta_{k}\right|^{2} E_{\mathrm{x}}[k]$, with $\left|\beta_{k}\right|^{2} \doteq h_{\mathrm{d}} G_{\mathrm{s}} h[k]$ and $h[k], G_{\mathrm{s}}$ and $h_{\mathrm{d}}$ the individual uplink, satellite and common downlink power gain, respectively. The noise power spectral density at the gateway is $N_{0}=h_{\mathrm{d}} G_{\mathrm{s}} N_{\mathrm{u}}+N_{\mathrm{d}}$, with $N_{\mathrm{u}}$ and $N_{\mathrm{d}}$ the uplink and downlink contributions.

The receiver performs typical SIC-based demodulation: de-spreading with $c_{k}[\cdot]$ and subsequent decoding for each successive user, followed by user packet re-modulation and cancellation before proceeding to the next user. All users employ the same encoder, with a Cyclic Redundancy Check (CRC) to enable error detection at the decoder. The decoder's PER characteristic is a function PER $[\Gamma]$ of the SINR at the output of the symbol de-spreader (denoted $\Gamma$ ), as at that point and with long spreading codes [16], multiple access interference may be assumed Gaussian. We adopt a SIC policy whereby a user is canceled if its packet has been correctly decoded and demodulation always progresses to the next user independently of previous decoding errors (user packet losses). We assume imperfect cancellation, after which an implementation-dependent residual fraction $\epsilon(\Gamma)$ of the user energy remains. Then, the SINR at the $k$-th SIC stage is,

$$
\Gamma_{k}=\frac{E_{s}[k]}{N_{0}+\frac{\theta}{N} \sum_{i=1}^{k-1} \epsilon\left[i \mid \Gamma_{i}\right] E_{s}[i]+\frac{\theta}{N} \sum_{i^{\prime}=k+1}^{K} E_{s}\left[i^{\prime}\right]}
$$

with $\epsilon\left[i \mid \Gamma_{i}\right]$ a binary random variable equal to 1 (packet loss) or to $\epsilon\left(\Gamma_{i}\right)$ (packet decoded) and $\theta$ an average decorrelation factor accounting for inter-user timing variations [12][15]. In the large-user limit, we define the continuous user index $t \doteq$ $\lim _{K \rightarrow \infty} \frac{k}{K}$, with $0 \leq t \leq 1$. We also let $N \rightarrow \infty$ so that the system load $\alpha \doteq \frac{K}{N}$ is held constant. Under these assumptions, we define: (i) the asymptotic continuous energy profile $E_{s}(t)$, with $E_{s}[k]$ obtained by sampling: $E_{s}[k]=E_{s}\left(\frac{k}{K}\right)$ and (ii) its corresponding asymptotic continuous SINR profile $\Gamma(t) \doteq$ $\lim _{K, N \rightarrow \infty} \Gamma_{k}$, expressed as,

$$
\begin{aligned}
& \Gamma(t)=\frac{E_{s}(t)}{N_{\mathrm{t}}(t)}=\frac{E_{s}(t)}{N_{0}+\xi_{\mathrm{prv}}(t)+\xi_{\mathrm{rem}}(t)} \\
& \xi_{\mathrm{prv}}(t)=\alpha \theta \int_{0}^{t} r(\Gamma(\tau)) \cdot E_{s}(\tau) \mathrm{d} \tau, \xi_{\mathrm{rem}}(t)=\alpha \theta I(t)
\end{aligned}
$$

with $N_{\mathrm{t}}(t)$ the noise plus interference term, and $\xi_{\mathrm{prv}}(t)$ and $\xi_{\text {rem }}(t)$ the interference from previous and remaining users, respectively, given by the following two definitions [15]:

$r(\Gamma) \doteq \epsilon(\Gamma)+(1-\epsilon(\Gamma)) \operatorname{PER}[\Gamma], I(t) \doteq \int_{t}^{1} E_{s}(\tau) \mathrm{d} \tau$

Expression (3) has been shown [13] equivalent to the following Ordinary Differential Equation (ODE) in $\Gamma(t)$ (given $E_{s}(t)$ ),

$$
\frac{\mathrm{d}}{\mathrm{d} t} \log E_{s}(t)=\frac{\mathrm{d}}{\mathrm{d} t} \log \Gamma(t)-\alpha \Phi[\Gamma(t)]
$$

with $\Phi[\Gamma] \doteq \theta(1-\epsilon(\Gamma)) \Gamma \cdot \operatorname{PSR}[\Gamma]$ and $\operatorname{PSR}[\Gamma] \doteq 1-\operatorname{PER}[\Gamma]$ the Packet Success Rate (PSR) function.

On the transmission side, we define the corresponding asymptotic profiles $E_{\mathrm{x}}(t)$ and $h(t)$, so that the received energy profile can be expressed as $E_{s}(t)=h_{\mathrm{d}} G_{\mathrm{s}} h(t) E_{\mathrm{x}}(t)$.

\section{OPTIMUM SINR AND ENERGY PROFILES}

We derive the SINR and energy profiles $\Gamma(t)$ and $E_{s}(t)$ that optimize the aggregate spectral efficiency achieved by the studied SIC receiver in the large-user regime. From the previous section, the corresponding transmitted energies are obtained $^{1}$ from $E_{\mathrm{x}}(t)=\left(h_{\mathrm{d}} G_{\mathrm{s}} h(t)\right)^{-1} E_{s}(t)$ and will be discussed further in Section IV. To enable power control, we assume that each user packet sent to the gateway contains its local (perfect) $h[k]$ estimate obtained from a downlink pilot signal (see Section I) and that the gateway broadcasts a compressed table $E_{h}(\mathrm{~h}) \doteq E_{x}\left(h^{-1}(\mathrm{~h})\right)$ to the users on a low-rate control channel. In this way, each user may set its symbol energy to $E_{h}(h[k])$ to generate the required $E_{s}(t)$.

First, we define an admissible energy profile $E_{s}(t)$ as one that fulfils the two following conditions in terms of the user variable $t$ and an admission parameter $t_{0}$,

(i) an admission constraint: $\left.E_{s}(t)\right|_{t_{0}<t \leq 1}=0$.

(ii) a finite differentiable non-zero energy constraint for each active user:

$$
0<\left.E_{s}(t)\right|_{0 \leq t \leq t_{0}}<\infty
$$

We are interested in optimizing the overall system spectral efficiency (bps/Hz), which is proportional to the average PSR:

$$
\overline{\mathrm{psr}} \doteq \int_{0}^{1} \operatorname{PSR}[\Gamma(t)] \mathrm{d} t
$$

Hence, for $E_{s}(t)$ an admissible profile, we seek to optimize $\overline{\mathrm{psr}}$ jointly over $E_{s}(t)$ (given $t_{0}$ ) and the admission parameter $t_{0}$, subject to an average energy constraint $\bar{E}_{\mathrm{s}}$ that sets the $\mathrm{OP}$ of the on-board amplifier ${ }^{2}$. Accordingly, the following constrained optimization problem needs to be solved:

$$
\begin{aligned}
& \overline{\mathrm{psr}}_{\max } \doteq \max _{0 \leq t_{0} \leq 1}\left[\max _{E_{s}(t)}\left[\int_{0}^{1} \operatorname{PSR}[\Gamma(t)] \mathrm{d} t\right]\right] \\
& \text { s.t. } E_{s}(t): \text { conditions }(\mathrm{i})-(\mathrm{ii}) \\
& \text { s.t. } \int_{0}^{1} E_{s}(t) \mathrm{d} t=I(0)=\bar{E}_{\mathrm{s}}
\end{aligned}
$$

where $\Gamma(t)$ in (9) is obtained from $E_{s}(t)$ by solving the ODE in (6). The inner maximization over $E_{s}(t)$ in (9) constitutes a VC problem (see Section III-A). The maximization over $t_{0}$, implicit in conditions (i)-(ii), constitutes instead a simpler univariate optimization problem.

${ }^{1}$ Given that the optimum $E_{s}(t)$ is non-increasing (Property 2 in Section III-A), $h(t)$ is taken as the non-increasing profile of channel power gains.

${ }^{2}$ Given the gain $G_{\mathrm{S}} h_{\mathrm{d}}$ and the uplink noise power level $N_{\mathrm{u}}$ (perfect pilotbased estimates assumed at the gateway), the signal bandwidth $W$ and chip rate $r_{c} \doteq 1 / T_{c}$, we have: $\mathrm{OP}=N_{\mathrm{u}} W+K \cdot \bar{E}_{s} r_{c} \cdot\left(G_{\mathrm{s}} h_{\mathrm{d}}\right)^{-1}$. 


\section{A. Solution to the VC problem and Invariance Equation}

Sections VI and VII show that the energy profile $E_{s, *}(t)$ and its resulting SINR profile $\Gamma_{*}(t)$ that achieve a local optimum to the inner VC problem in (9) verify Properties 1 and 2:

Property 1: Conditions for local optimality (Section VI-A). If $\Gamma_{*}(t)>\Gamma_{\mathrm{ip}}, \forall t \in\left[0, t_{0}\right]$, with $\Gamma_{\mathrm{ip}}$ the inflection point of the PSR function $\left(\mathrm{PSR}^{\prime \prime}\left[\Gamma_{\mathrm{ip}}\right]=0\right)$, then, $\Gamma_{*}(t)$ yields a local optimum. Otherwise, $\Gamma_{*}(t)$ constitutes a saddle point: this occurs when $\Gamma_{*}(t)<\Gamma_{\text {ip }}$ over a sub-interval within $\left[0, t_{0}\right]$.

Property 2: The solution profiles $\Gamma_{*}(t)$ and $E_{s, *}(t)$ are both non-increasing in $t$. This result, proved in Section VII, is subject to the monotonicity conditions exposed therein.

We present now a system of nonlinear and differential equations whose solution constitutes a stationary point to the inner VC problem in (9). This system is established in terms of the profiles $E_{s}(t), \Gamma(t)$ and $I(t)$ for $0 \leq t \leq t_{0}$ as follows,

1) First Equation: the integrated energy profile $I(t)$ in (5),

$$
\frac{\mathrm{d}}{\mathrm{d} t} I(t)=-E_{s}(t)
$$

2) Second Equation: SIC operation. See equation (6).

3) Third Equation: the Invariance Equation (IE). The first two system equations are fulfiled for each possible profile. The IE (Section VI), though, is specific to the stationary point and establishes an invariance throughout the SIC stages,

$$
\frac{\operatorname{PSR}^{\prime}[\Gamma(t)]}{N_{\mathrm{t}}(t)-\alpha I(t) \cdot \Phi^{\prime}[\Gamma(t)]}=\rho>0
$$

with $0 \leq t \leq t_{0}, \rho$ a constant value and $N_{\mathrm{t}}(t)=E_{s}(t) / \Gamma(t)$ the noise plus interference profile defined in (3).

The previous system must fulfil the following three boundary conditions given $\bar{E}_{\mathrm{s}}$ and $\rho$ (temporarily assumed known),

1) Boundary condition for $I(t)$ : we set $I(0)=\bar{E}_{\mathrm{s}}$.

2) Boundary condition for $\Gamma(t)$ : applying the IE at $t=0$, we set $\Gamma(0)=\Gamma_{0}$ by solving for $\Gamma_{0}$ in,

$$
\rho=\operatorname{PSR}^{\prime}\left[\Gamma_{0}\right] /\left(N_{0}+\alpha \theta \bar{E}_{\mathrm{s}}-\alpha \bar{E}_{\mathrm{s}} \cdot \Phi^{\prime}\left[\Gamma_{0}\right]\right)
$$

where, from (3), we have used $N_{\mathrm{t}}(0)=N_{0}+\xi_{\text {prv }}(0)+\xi_{\text {rem }}(0)$, with $\xi_{\mathrm{prv}}(0)=0$ (from [15]) and $\xi_{\mathrm{rem}}(0)=\alpha \theta \bar{E}_{\mathrm{s}}$ (from (5)).

3) Boundary condition for $E_{s}(t)$ : from (3), we set $t=0$ and we get that $E_{s}(0)=N_{\mathrm{t}}(0) \cdot \Gamma_{0}=\left(N_{0}+\alpha \theta \bar{E}_{\mathrm{s}}\right) \cdot \Gamma_{0}$.

Solution procedure: For each possible $\Gamma_{0}$ and its respective $\rho$ in (14), we solve the system for $E_{s}(t), \Gamma(t)$ and $I(t)$ by a standard numerical procedure based on Euler's method for differential equations, with the boundary conditions as initialization at $t=0$. A valid $t_{0}$ for each $\Gamma_{0}$ is computed from $I\left(t_{0}\right)=0$ (when $t_{0} \leq 1$ ) and its PSR obtained to perform the outer univariate maximization in (9). The maximizing $t_{0}$ yields the corresponding SINR and energy profiles.

\section{Simulations}

We evaluate the previous theoretical results in a representative interference-dominated scenario featuring non-orthogonal spread-spectrum users with spreading factor $N=2048$ and a received average $\bar{E}_{s} / N_{0}$ of $15 \mathrm{~dB}$. The SIC residual interference power factor is assumed independent of the SINR, with $\epsilon(\Gamma)=0.1$. The decorrelation factor is set to $\theta=1$.
As the optimum SINR and energy profiles in Section III depend on the considered modulation and forward-error correcting (FEC) code, we have evaluated the SIC performance for QPSK and two representative FEC codes (Fig. 1): (a) the classical convolutional code (CC) of rate $1 / 2$ and generator polynomials $133_{\text {oct }}$ and $171_{\text {oct }}$ (adopted in standards such as DVB-S or IEEE 802.11), with a packet length of 320 information bits; (b) the DVB-RCS turbo-code (TC) with rate $1 / 2$ using max-log-MAP decoding, with a packet length of 864 information bits. The TC is selected as an instance of an abrupt PER $[\Gamma]$ curve, whereas the $\mathrm{CC}$ is used to evaluate the performance for a smoother $\operatorname{PER}[\Gamma]$ curve. Property 1 (Section III) shows that the optimum SINR profile $\Gamma_{0}(t)$ must be above a certain threshold $\Gamma_{\text {ip }}$ for any $0 \leq t \leq t_{0}$. To check this condition, the PSR inflection point is indicated in Fig. 1, for the two FEC codes, with a vertical dashed line.

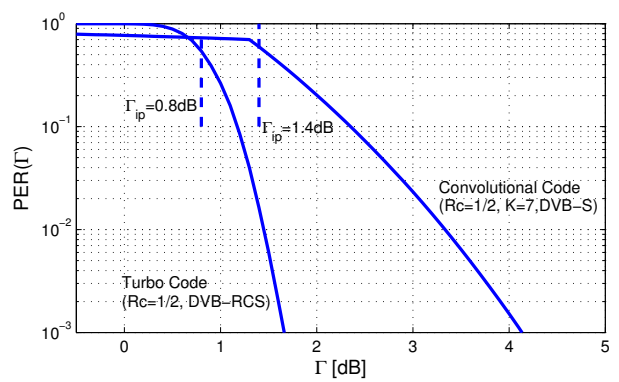

Fig. 1. PER vs. SINR curves for the two studied FEC codes.

Fig. 2 depicts, in the upper plot, the system's spectral efficiency (SE) $2 \alpha R_{c} \overline{\mathrm{psr}}_{\max }$ versus the load $\alpha=\frac{K}{N}$ for the $\mathrm{CC}$, with $R_{c}$ the code rate. As shown, the SE does not collapse for high loads, but holds its performance when $\alpha$ exceeds a maximum value saturating the system. The bottom plot in Fig. 2 shows the value of $t_{0}$ that maximizes (9) and the evolution of the range of $\Gamma(t)$ in terms of $\alpha$. The range of $\Gamma(t)$ is defined for each $\alpha$ by its initial (maximum) and last (minimum) SINR values $\Gamma(0)$ and $\Gamma\left(t_{0}\right)$, resp., since $\Gamma(t)$ (Property 2) is nonincreasing. As shown, when the system is underloaded, the SINR profile $\Gamma(t)$ tends to be uniform with $t_{0}=1$. On the contrary, when approaching saturation, the optimum SINR profile is no longer uniform, while $t_{0}$ still satisfies $t_{0}=1$. When the traffic load exceds the saturation value, the range of $\Gamma(t)$ does not increase but $t_{0}$ starts decreasing and regulates the system load. This behavior guarantees that the SINR profile satisfies $\Gamma(t)>\Gamma_{\mathrm{ip}}$.

In Fig. 3, a $13 \%$ overloaded system $(\alpha=1.3)$ handling approximately $K=2662$ users is simulated to complement the results in Fig. 2, evidencing that the SINR profile that maximizes (9) in not uniform. A Monte Carlo simulation with 500 runs shows the average SINR profile (circles) obtained from the optimum symbol-energy profile (middle plot). The solid line corresponds to the theoretical optimum profiles.

The same curves are plotted in Figs. 4 and 5 for the more powerful TC, showing a much higher admissible load. The maximum accepted load for the TC is approx. $\alpha=1.67(K \approx$ 

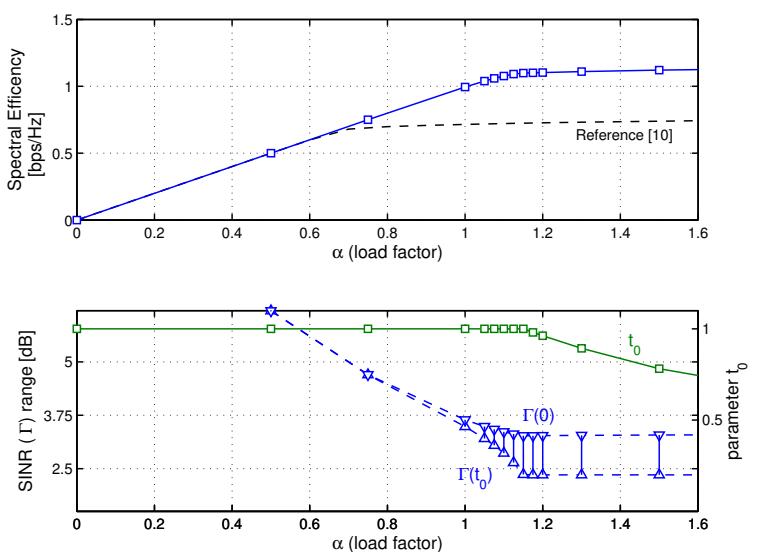

Fig. 2. Spectral efficiency vs. traffic load $\alpha$ (above) and its associated $\Gamma(t)$ range and $t_{0}$ (bottom) for the conv. code. The marks $\nabla$ indicate $\Gamma(0)=\max \{\Gamma(t)\}$, and the marks $\Delta$ indicate $\Gamma\left(t_{0}\right)=\min \{\Gamma(t)\}$.
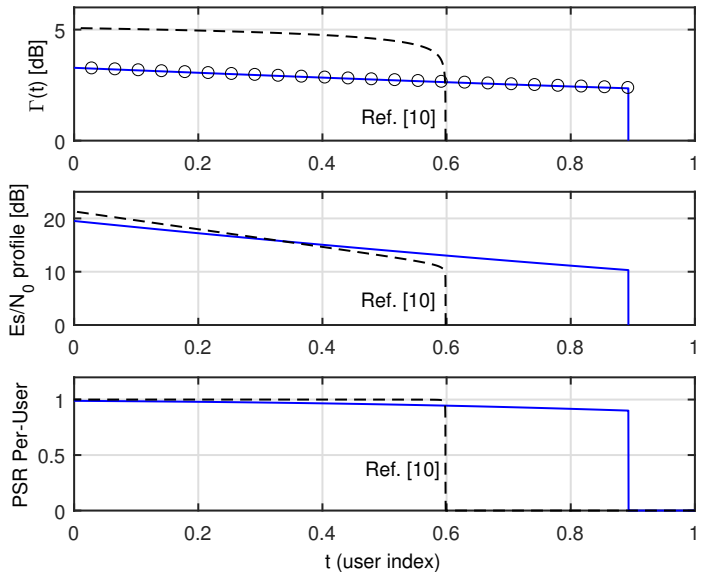

Fig. 3. Per-user profiles vs. user index $t$ for a $13 \%$ overloaded system $(\alpha=1.3, K \approx 2662)$ for the convolutional code.

3420 users) vs. $\alpha=1.15$ ( $K \approx 2355$ users) for the $\mathrm{CC}$. In both cases, the $\mathrm{SE}$ exceeds $1 \mathrm{bps} / \mathrm{Hz}$, the limit value for orthogonal multiple access. As shown in Fig.4, the range of $\Gamma(t)$ is reduced for the TC due to its steeper PER $[\Gamma]$ curve.

In Figs. 2-5, we also simulate the SIC policy in [11], which stops iterative decoding when the first packet error occurs. To avoid this situation, the admitted load is severely limited and, thus, the system SE degrades (e.g., 34\% with the CC).

Fig. 6 complements the PSR information in Figs. 3 and 5 (bottom). It shows, for the $\mathrm{CC}$ and the TC, the evolution of the corresponding PER (average over the 500 Monte Carlo runs) across the SIC stages, in comparison with the theoretical prediction (PSR profiles in the mentioned figures). In this respect, accurate results for the average PER using Equation (6) were reported in [15] for $K \geq 512$ users.
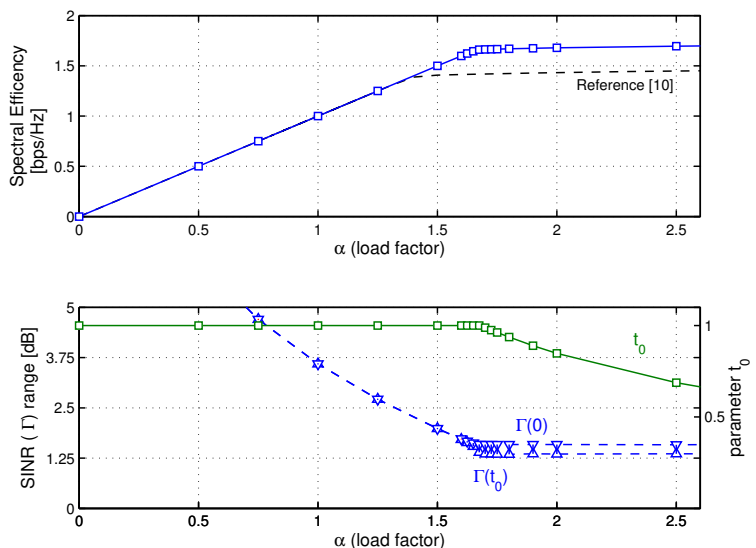

Fig. 4. Spectral efficiency vs. traffic load $\alpha$ (above) and its associated $\Gamma(t)$ range and $t_{0}$ (bottom) for the turbo code. The marks $\nabla$ indicate $\Gamma(0)=\max \{\Gamma(t)\}$, and the marks $\Delta$ indicate $\Gamma\left(t_{0}\right)=\min \{\Gamma(t)\}$.
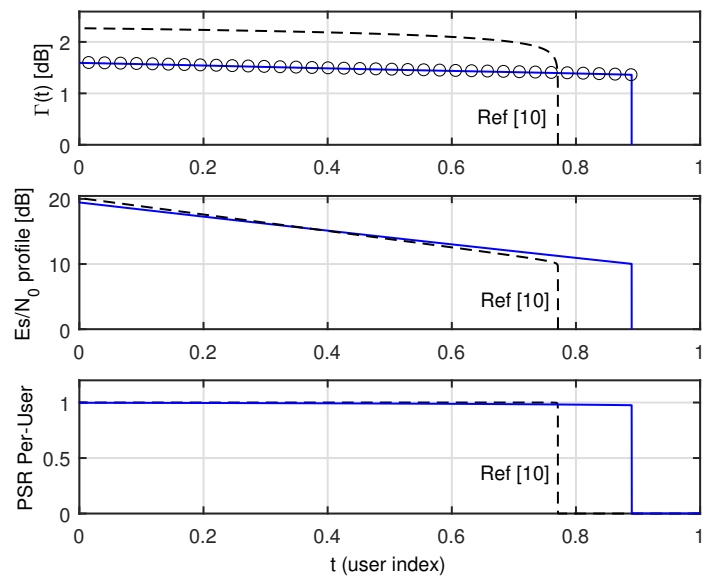

Fig. 5. Per-user profiles vs. user index $t$ for a $13 \%$ overloaded system $(\alpha=1.9, K \approx 3891)$ for the turbo code.

Finally, Fig. 7 shows the transmission profile $E_{\mathrm{x}}(t) / N_{0}$ for three uplink channel power gain profiles $h(t)$. We have used an exponential $h(t)=h_{0} e^{-R_{0}^{2} t / \sigma_{r}^{2}}$ to model a circular coverage area of radius $R_{0}$ with uniformly distributed users and an ideal Gaussian beamshaped pathloss $P(r)$ versus radius, for which $h(t)=P\left(R_{0} \sqrt{t}\right)$. Results have been obtained with a variation range $L=e^{R_{0}^{2} / \sigma_{r}^{2}}$ of $5 / 10 / 15 \mathrm{~dB}$ within this area, corresponding to the reception profile $E_{s}(t) / N_{0}$ in Fig. 5 for the overload regime. The user power imbalance at transmission,

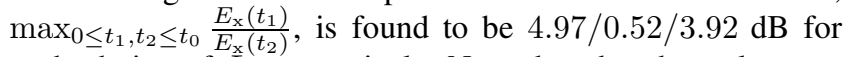
each choice of $L$, respectively. Note that the channel power gain profile contributes to reducing the power imbalance at transmission with respect to the required $9.42 \mathrm{~dB}$ in reception (Fig. 5 (middle)). In fact, depending on the specific form of $h(t)$ (i.e., $L=15 \mathrm{~dB}$ ), a non-decreasing profile may result. 


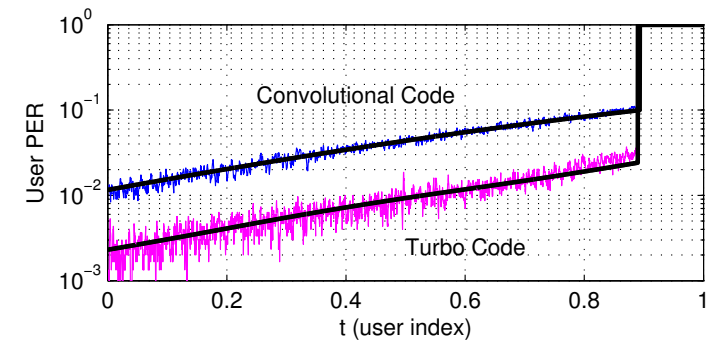

Fig. 6. PER profile of the CC (resp. TC) across the SIC stages, corresponding to the theoretical PSR profile in Fig. 3 (resp. Fig. 5) (blue/magenta) Monte Carlo average over 500 SIC runs with local smoothing over 6 consecutive users; (black) theoretical prediction.

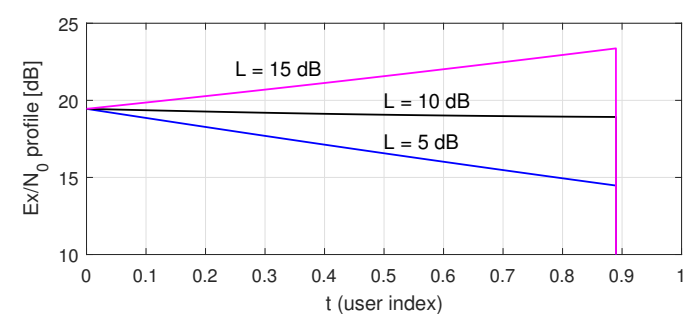

Fig. 7. Transmitted energy profiles normalized to $N_{0}$, for a reference satellite link gain constraint $h_{\mathrm{d}} G_{\mathrm{s}} h(0)=1$ and the parameter settings for the overloaded scenario in Fig. 5 (TC).

\section{CONCLUSIONS}

Variational Calculus constitutes a powerful tool to optimize advanced interference cancellation architectures enabling massive non-orthogonal multiple access. We have used it to derive the user energy profile that maximizes the spectral efficiency of a SIC receiver handling a large number of coded spreadspectrum users. In contrast to information theoretic works [12], we have assumed practical modulation and coding schemes. An analysis of the numerical results has shown that:

(i) the optimum SINR profile is a decreasing function of the SIC decoding order (this differs from capacity studies in which the optimum SINR profile is found to be uniform [12]). Hence, different users are subject to different PERs (Fig. 6).

(ii) all users are active while the system is underloaded. However, if the load exceeds the saturation level, the admission parameter $t_{0}$ is activated in order to regulate the admitted load.

\section{ApPEndix I: Stationary Point Equation}

Let us consider infinitesimal SINR variations $\delta \Gamma(t) \doteq a v(t)$ on a (constrained) SINR profile $\Gamma_{0}(t)$. The infinitesimal term $a \rightarrow 0$ sets, given $v(t)$, the magnitude of $\delta \Gamma(t) . v(t)$ is admissible if the corresponding $E_{s}(t)$ is admissible and fulfils the problem constraints. Let $\Gamma_{0}(t)$ be a stationary point of the VC problem in (9). Then, for $\Gamma(t)=\Gamma_{0}(t)+a v(t), 0 \leq t \leq t_{0}$, we construct the following Lagrangian with respect to $a$,

$$
\mathcal{L}(a) \doteq \int_{0}^{t_{0}} \operatorname{PSR}[\Gamma(t)] \mathrm{d} t-\lambda \cdot\left(\int_{0}^{t_{0}} E_{s}(t) \mathrm{d} t-\bar{E}_{\mathrm{s}}\right)
$$

Let $\mathcal{V}$ denote the set of admissible SINR variations $v(t)$. Thus, for $\Gamma_{0}(t)$ to be a stationary point of (15), we must have that,

$$
\lim _{a \rightarrow 0} \nabla_{a}[\mathcal{L}(a)]=0 \quad, \quad \forall v(t) \in \mathcal{V}
$$

Applying the gradient $\nabla_{a}$ to (15), the first term yields,

$$
\nabla_{a} \int_{0}^{t_{0}} \operatorname{PSR}[\Gamma(t)] \mathrm{d} t=\int_{0}^{t_{0}} \operatorname{PSR}^{\prime}[\Gamma(t)] v(t) \mathrm{d} t
$$

Let us look at the second term. First, from (6), integrating with respect to $t$ on both sides and using $N_{\mathrm{t}}(t)$ in (3), we have,

$$
E_{s}(t)=\Gamma(t) \cdot N_{\mathrm{t}}(0) e^{-B(t)}, B(t) \doteq \alpha \int_{0}^{t} \Phi[\Gamma(\tau)] \mathrm{d} \tau
$$

where, using (3)-(5): $N_{\mathrm{t}}(0)=E_{s}(0) / \Gamma(0)=N_{0}+\alpha \theta \bar{E}_{\mathrm{s}}$ is independent of $a$. Applying $\nabla_{a}$ to the second term in (15),

$$
\begin{gathered}
\nabla_{a} \int_{0}^{t_{0}} E_{s}(t) \mathrm{d} t=N_{\mathrm{t}}(0) \int_{0}^{t_{0}} e^{-B(t)} v(t) \mathrm{d} t \\
+\quad N_{\mathrm{t}}(0) \int_{0}^{t_{0}} \Gamma(t) e^{-B(t)}\left(-\alpha \int_{0}^{t} \Phi^{\prime}[\Gamma(\tau)] v(\tau) \mathrm{d} \tau\right) \mathrm{d} t
\end{gathered}
$$

Using (18), the previous right-hand side term becomes,

$\int_{0}^{t_{0}} N_{\mathrm{t}}(t) v(t) \mathrm{d} t-\int_{0}^{t_{0}} \alpha E_{s}(t) \int_{0}^{t_{0}} \Phi^{\prime}[\Gamma(\tau)] v(\tau) \mathrm{u}(t-\tau) \mathrm{d} \tau \mathrm{d} t$ for $\mathrm{u}(\tau)$ the unit step function. Exchanging integrals over $t, \tau$ in the second term, and as $E_{s}(t)=0$ for $t_{0}<t \leq 1$, we get $I(t)$ in (5): $\int_{0}^{t_{0}} E_{s}(t) \mathrm{u}(t-\tau) \mathrm{d} t=\int_{\tau}^{t_{0}} E_{s}(t) \mathrm{d} t=I(\tau)$. Thus, using $t$ as in integration variable, we finally obtain,

$$
\nabla_{a} \int_{0}^{t_{0}} E_{s}(t) \mathrm{d} t=\int_{0}^{t_{0}}\left(N_{\mathrm{t}}(t)-\alpha I(t) \Phi^{\prime}[\Gamma(t)]\right) v(t) \mathrm{d} t
$$

Let $D(t) \doteq N_{\mathrm{t}}(t)-\alpha I(t) \Phi^{\prime}[\Gamma(t)]$. Combining (20) with (17) in (16), we get the stationary point equation for all $v(t) \in \mathcal{V}$,

$$
\lim _{a \rightarrow 0} \int_{0}^{t_{0}}\left(\operatorname{PSR}^{\prime}[\Gamma(t)]-\lambda \cdot D(t)\right) v(t) \mathrm{d} t=0
$$

It only remains now to determine $\mathcal{V}$ : as the $\bar{E}_{\mathrm{s}}$ constraint must be fulfiled along the variation, this is equivalent to setting,

$$
\lim _{a \rightarrow 0} \nabla_{a}\left[\int_{0}^{t_{0}} E_{s}(t) \mathrm{d} t-\bar{E}_{\mathrm{s}}\right]=0
$$

At this point, we drop $\lim _{a \rightarrow 0}$ and interpret $\Gamma(t), E_{s}(t), I(t)$ and $N_{\mathrm{t}}(t)$ to correspond to the stationary point. The gradient in (22) appears in (20). Hence, any admissible $v(t)$ at any $\Gamma(t)$ must fulfil: $\int_{0}^{t_{0}}\left(N_{\mathrm{t}}(t)-\alpha I(t) \Phi^{\prime}[\Gamma(t)]\right) v(t) \mathrm{d} t=0$, which, combined with (21), defines $\mathcal{V}$ at a stationary point $\Gamma(t)$ as,

$$
\mathcal{V} \doteq\left\{v(t): \int_{0}^{t_{0}} \operatorname{PSR}^{\prime}[\Gamma(t)] \cdot v(t) \mathrm{d} t=0\right\}
$$

Hence, if (21) must hold for any $v(t) \in \mathcal{V}$, then, from (23), the factor of $v(t)$ in (21) must be either zero or proportional to $\operatorname{PSR}^{\prime}[\Gamma(t)]$. This finally establishes, for $q \neq 1$, that: $\operatorname{PSR}^{\prime}[\Gamma(t)]-\lambda \cdot D(t)=q \operatorname{PSR}^{\prime}[\Gamma(t)]$. Setting $\rho \doteq \frac{\lambda}{1-q}$, we get the Invariance Equation in (13) along the SIC stages, where $\rho>0$ as $I\left(t_{0}\right)=0$. Note that setting $q=1$ leads to a contradiction at $t=t_{0}$, where $I\left(t_{0}\right)=0 \Rightarrow D\left(t_{0}\right)=N_{\mathrm{t}}\left(t_{0}\right)=0$, but necessarily $N_{\mathrm{t}}(t)>0$, from $N_{\mathrm{t}}(t)=N_{\mathrm{t}}(0) e^{-B(t)}$ in (18). 


\section{A. Extremality Analysis}

We derive conditions for $\Gamma_{0}(t)$ to be a local maximum. We expand the $\overline{\mathrm{psr}}$ around $\Gamma_{0}(t)$ up to second order in $a$,

$$
\overline{\mathrm{pSr}}=\sum_{i=0}^{2} \int_{0}^{t_{0}} \operatorname{PSR}^{(i)}\left[\Gamma_{0}(t)\right] \frac{(a v(t))^{i}}{i !} \mathrm{d} t+o\left(a^{2}\right)
$$

Clearly, using (23), for $v(t)$ admissible at the stationary point $\Gamma_{0}(t)$, the $i=1$ term above in $a v(t)$ is exactly zero and,

$$
\overline{\mathrm{pSr}} \simeq \int_{0}^{t_{0}} \operatorname{PSR}\left[\Gamma_{0}(t)\right] \mathrm{d} t+\int_{0}^{t_{0}} \operatorname{PSR}^{\prime \prime}\left[\Gamma_{0}(t)\right] \cdot \frac{a^{2} v^{2}(t)}{2} \mathrm{~d} t
$$

Thus, $D_{2} \doteq \int_{0}^{t_{0}} \mathrm{PSR}^{\prime \prime}\left[\Gamma_{0}(t)\right] v^{2}(t) \mathrm{d} t$ must be negative for all $v(t) \in \mathcal{V} . D_{2}<0$ holds when $\Gamma_{0}(t)>\Gamma_{\text {ip }}$ at $0 \leq t \leq t_{0}$, with $\Gamma_{\text {ip }}$ the inflection point of $\operatorname{PSR}[\Gamma]$, at which $\operatorname{PSR}^{\prime \prime}\left[\Gamma_{\text {ip }}\right]=$ 0 . But, if for $\mathcal{T} \doteq\left[t_{1}, t_{2}\right] \subset\left[0, t_{0}\right]$ we have $\Gamma_{0}(t) \leq \Gamma_{\text {ip }}$, then: $\operatorname{PSR}^{\prime \prime}\left[\Gamma_{0}(t)\right] \geq 0$ for $t \in \mathcal{T}$. In that case, consider a variation $v_{*}(t)=0, t \notin \mathcal{T}$ that still fulfils (23). Hence, $v_{*}(t)$ is admissible but yields $D_{2} \geq 0$. Thus, for such $\mathcal{T}$ and $v(t) \in$ $\mathcal{V}$ at $\Gamma_{0}(t)$, we may have $D_{2}<0$ (when $v(t)=0$ where $\mathrm{PSR}^{\prime \prime}\left[\Gamma_{0}(t)\right] \geq 0$ ) or $D_{2} \geq 0$ (when $v(t)=v_{*}(t)$ ). Thus, a $\Gamma_{0}(t)$ that at some $\mathcal{T}$ fulfils $\Gamma_{0}(t)<\Gamma_{\text {ip }}$, is a saddle point. We must have $\Gamma_{0}(t)>\Gamma_{\text {ip }}$ over $0 \leq t \leq t_{0}$ for a local maximum.

\section{Appendix II: Monotonicity of $E_{s}(t)$ And $\Gamma(t)$}

From $I(t)$ in (5) and $N_{\mathrm{t}}(t)$ in (3), we get that $\frac{\mathrm{d}}{\mathrm{d} t} I=-N_{\mathrm{t}} \cdot \Gamma$. Hence, substituting $N_{\mathrm{t}}(t)=E_{s}(t) / \Gamma(t)$ into (6), we get,

$$
\frac{\mathrm{d}}{\mathrm{d} t} N_{\mathrm{t}}=\alpha(\Phi[\Gamma] / \Gamma) \cdot \frac{\mathrm{d}}{\mathrm{d} t} I
$$

Now, from (13), we solve for $N_{\mathrm{t}}$ versus $\Gamma$ and $I$ and obtain that: $N_{\mathrm{t}}(\Gamma, I)=\rho^{-1} \cdot \mathrm{PSR}^{\prime}[\Gamma]+\alpha I \cdot \Phi^{\prime}[\Gamma]$, with differential $\mathrm{d} N_{\mathrm{t}}=\left(\partial_{\Gamma} N_{\mathrm{t}}\right) \cdot \mathrm{d} \Gamma+\left(\partial_{I} N_{\mathrm{t}}\right) \cdot \mathrm{d} I$.

Let $\Gamma_{I}(I)$ map $I(t)$ to SINR, with $\Gamma(t)=\Gamma_{I}(I(t))$. Here, $\Gamma_{I}(I)$ is well-defined as $I(t)$ is non-increasing in $t$. Computing the partial derivatives in $\partial_{\Gamma} N_{\mathrm{t}}$ and $\partial_{I} N_{\mathrm{t}}$ and using (26) for $\mathrm{d} N_{\mathrm{t}}$, we get the following ODE for $\Gamma_{I}(I)$, where $\rho>0$,

$$
\frac{\mathrm{d} \Gamma_{I}}{\mathrm{~d} I}=\Psi_{\alpha, \rho}\left(\Gamma_{I}, I\right) \doteq \frac{\left(\alpha / \Gamma_{I}\right)\left(\Phi\left[\Gamma_{I}\right]-\Gamma_{I} \Phi^{\prime}\left[\Gamma_{I}\right]\right)}{\rho^{-1} \operatorname{PSR}^{\prime \prime}\left[\Gamma_{I}\right]+\alpha I \cdot \Phi^{\prime \prime}\left(\Gamma_{I}\right)}
$$

Monotonicity conditions (SINR): $\Gamma_{I}(I)$ is a monotone function if $\Psi_{\alpha, \rho}\left(\Gamma_{I}, I\right)$ in (27) does not change its sign. This is fulfiled for typical PSR $[\Gamma]$ functions over some SINR threshold: $\Gamma \geq \Gamma_{\text {th }}$, where the inequalities $\operatorname{PSR}^{\prime \prime}[\Gamma]<0$, $\Phi^{\prime \prime}[\Gamma]<0$ and $1-\Gamma \cdot \frac{\mathrm{d}}{\mathrm{d} \Gamma} \log \Phi[\Gamma]<0$ hold simultaneously. Hence, $\Psi_{\alpha, \rho}\left(\Gamma_{I}, I\right) \geq 0$ and thus, from (27), $\Gamma_{I}(I)$ is nondecreasing in $I: \frac{\mathrm{d}}{\mathrm{d} I} \Gamma_{I} \geq 0$. In consequence, $\Gamma(t)=\Gamma_{I}(I(t))$ is non-increasing in $t$.

Now, let us define $E_{I}: I \rightarrow E_{s}$, with $E_{s}(t)=E_{I}(I(t))$.

Monotonicity conditions (Energy): $E_{I}(I)$ is monotone. Let $\Gamma_{*}(I)$ solve (27). Then, $E_{I}(I)=\Gamma_{*}(I) N_{\mathrm{t}}\left(\Gamma_{*}(I), I\right) \Rightarrow$ $\frac{\mathrm{d}}{\mathrm{d} I} E_{I}=N_{\mathrm{t}} \frac{\mathrm{d}}{\mathrm{d} I} \Gamma_{I}+\Gamma_{I} \frac{\mathrm{d}}{\mathrm{d} I} N_{\mathrm{t}}$. As $\frac{\mathrm{d}}{\mathrm{d} I} \Gamma_{I} \geq 0$, and, from (26), $\Phi[\Gamma] \geq 0$, we get $\frac{\mathrm{d}}{\mathrm{d} I} N_{\mathrm{t}} \geq 0$. Thus, $\frac{\mathrm{d}}{\mathrm{d} I} E_{I} \geq 0$. Hence, $\frac{\mathrm{d}}{\mathrm{d} I} E_{I}(I) \geq 0$ and $E_{s}(t)=E_{I}(I(t))$ is non-increasing in $t$.

\section{ACKNOWLEDGMENT}

Work supported by projects TEC2013-47020-C2-2-R (COMPASS) and TEC2016-76409-C2-1-R (AEI/FEDER, UE) (WINTER): Ministerio de Economía, Industria y Competitividad (Spanish administration); 2014 SGR 60: AGAUR (Catalan administration).

\section{REFERENCES}

[1] P. Patel and J. Holtzman, "Analysis of a simple successive interference cancellation scheme in a DS/CDMA system," IEEE Journal on Selected Areas in Communications, vol. 12, no. 5, pp. 796 - 807, June 1994.

[2] Y. Yingqun and G. Giannakis, "High-throughput random access using successive interference cancellation in a tree algorithm," IEEE Trans. on Information Theory, vol. 53, no. 12, pp. 4628 - 4639, Dec. 2007.

[3] Y. Hu, W. Yang, and Y. Cai, "Throughput analysis of slotted ALOHA with cooperative transmission using successive interference cancellation," Science in China Series F: Information Sciences (Springer), vol. 52, no. 12, pp. $2354-2359,2009$.

[4] O. del Río Herrero and R. de Gaudenzi, "High efficiency satellite multiple access scheme for machine-to-machine communications," IEEE Trans. on Aerospace and Electronics Engineering, vol. 48, no. 4, pp. 2961 - 2989, Oct. 2012.

[5] G. Gallinaro, N. Alagha, R. De Gaudenzi, K. Kansanen, R. Muller, and P. Rossi, "ME-SSA: An advanced random access for the satellite return channel," in 2015 IEEE Intl. Conf. on Communications (ICC), June 2015, pp. 856-861.

[6] S. Scalise, C. Niebla, R. De Gaudenzi, O. del Río Herrero, D. Finocchiaro, and A. Arcidiacono, "S-MIM: a novel radio interface for efficient messaging services over satellite," IEEE Communications Magazine, vol. 51, no. 3, pp. 119-125, March 2013.

[7] E. Casini, R. de Gaudenzi, and O. del Río Herrero, "Contention resolution diversity slotted ALOHA (CRDSA): An enhanced random access scheme for satellite access packet networks," IEEE Trans. on Wireless Communications, vol. 6, no. 4, pp. 1408 - 1417, April 2007.

[8] G. Liva, "Graph-based analysis and optimization of contention resolution diversity slotted ALOHA," IEEE Trans. on Communications, vol. 59, no. 2, pp. 477-487, Feb. 2011.

[9] P. Salvo Rossi, K. Kansanen, R. Muller, and C. Rachinger, "Power randomization for iterative detection over random-access fading channels," IEEE Trans. on Wireless Communications, vol. 14, no. 10, pp. 57045713, Oct 2015.

[10] International Telecommunications Union (ITU), Radiocommunication Bureau, BR Workshop on the efficient use of the spectrum/orbit resource, May, 2009, Geneva.

[11] J. Sala-Alvarez, J. Villares, and F. Rey, "SINR profile for spectral efficiency optimization of SIC receivers in the many-user regime," in Proc. IEEE ICC 2015 Workshop on Massive Uncoordinated Access Protocols (MASSAP'15), London, U.K., Jun. 2015.

[12] D. Djonin and V. K. Bhargava, "Asymptotic analysis of the conventional decision feedback receiver in fading channels," IEEE Trans. on Wireless Communications, vol. 2, no. 5, pp. 1066 - 1078, May 2003.

[13] J. Sala, F. Rey, and J. Villares, "Asymptotically optimum energy profile for successive interference cancellation in DS-CDMA under a power unbalance constraint," IEEE Communications Letters, vol. 16, no. 2, pp. 172 - 175, Feb. 2012.

[14] F. Collard and R. De Gaudenzi, "On the optimum packet power distribution for spread ALOHA packet detectors with iterative successive interference cancellation," IEEE Trans. on Wireless Communications, vol. 13, no. 12, pp. 6783-6794, Dec 2014.

[15] J. Sala, J. Villares, and F. Rey, "Asymptotic and finite-user PER analysis of successive interference cancellation for DS-CDMA," IEEE Communications Letters, vol. 15, no. 11, pp. 1145 - 1147, Nov. 2011.

[16] S. Verdú and S. Shamai, "Spectral efficiency of CDMA with random spreading," IEEE Trans. on Information Theory, vol. 45, no. 2, pp. 622 - 640, Feb. 1999. 